\title{
Questes
}

vestes Revue pluridisciplinaire d'études médiévales

18 | 2010

La règle du jeu

\section{Les échecs et la cité de Troie}

\section{Anne Rochebouet et Anne Salamon}

\section{OpenEdition}

\section{Journals}

Édition électronique

URL : http://journals.openedition.org/questes/658

DOI : 10.4000/questes. 658

ISSN : 2109-9472

\section{Éditeur}

Les Amis de Questes

\section{Édition imprimée}

Date de publication : 15 février 2010

Pagination : $30-43$

ISSN : 2102-7188

\section{Référence électronique}

Anne Rochebouet et Anne Salamon, «Les échecs et la cité de Troie», Questes [En ligne], 18 | 2010, mis en ligne le 01 janvier 2014, consulté le 19 avril 2019. URL : http://journals.openedition.org/ questes/658; DOI : 10.4000/questes.658 


\title{
Les échecs et la cité de Troie
}

\author{
Anne Rochebouet et Anne Salamon
}

Plusieurs récits mythiques concernant l'origine des échecs circulent au Moyen Âge. Bien que leur genèse orientale fût connue des hommes médiévaux, ces derniers, pour leur assurer un prestige et une légitimité plus grande, ont lié leur invention à l'Antiquité, biblique ou classique. Salomon, poussé par le désir d'impressionner la reine de Saba, en est alors le plus souvent le créateur, mais Jacques de Cessoles ${ }^{1}$ en fait un jeu conçu pour éduquer Evilmerodag, fils de Nabuchodonosor, tandis que dans d'autres textes encore, c'est Aristote qui aurait instruit grâce à eux le jeune Alexandre le Grand. Christine de Pizan² attribue de son côté la paternité du jeu à Ulysse : l'« homme aux mille ruses » l'aurait inventé sous les murs de Troie pour divertir l'armée grecque. Selon une autre version, c'est Palamède, autre guerrier grec, déjà inventeur mythique de l'alphabet, qui l'aurait conçu ${ }^{3}$. Dans les textes non centrés sur la guerre de Troie mais où peuvent apparaître des personnages troyens, le jeu naît de l'ennui éprouvé lors du siège, mais surtout, il est une invention grecque. Or, dans la quasitotalité des textes de la matière troyenne médiévale, la création du jeu se fait à l'intérieur de la cité et constitue un exemple de l'excellence en tout domaine de la civilisation phrygienne, reflétant le parti-pris pro-troyen du Moyen Âge. La perception des jeux y évolue cependant au fil des

\footnotetext{
${ }^{1}$ De Ludo scacchorum, Ernst KöPKE (éd.), Jahresbericht der Mittheilungen aus den Handschriften der Ritter-Akademie zu Brandenburg, II, Brandenburg, 1879. Pour une traduction médiévale, voir JACQUES DE CESSOLES, Le Jeu des eschaz moralisé, traduction de Jean Ferron (1347), Alain Collet (éd.), Paris, Honoré Champion, « Classiques français du Moyen Âge », 1999.

${ }^{2}$ Voir illustration 1, Epistre Othea, $\mathrm{xv}^{\mathrm{e}}$ siècle (BnF, fr. 606, $\left.\mathrm{f}^{\circ} 39\right)$.

${ }^{3}$ Cette légende, transmise par plusieurs auteurs de l'Antiquité tardive (Cassiodore, Pausanias, ou encore saint Grégoire de Naziance) est assez répandue au Moyen Âge, comme en atteste la création, dans le Tristan en prose, du personnage de Palamède, qui enseigne le jeu à ses compagnons et dont les armes sont échiquetées d'argent et de sable.
} 
différentes adaptations, depuis le texte fondateur de Benoît de SainteMaure jusqu'aux compilations de la fin du Moyen Âge.

\section{Le Roman de Troie}

Écrit vers 1165 par Benoît de Sainte-Maure, un clerc tourangeau au service d'Henri II Plantagenêt, le Roman de Troie ${ }^{4}$ est l'œuvre en langue française du XII ${ }^{e}$ siècle qui nous est conservée dans le plus de manuscrits (trente complets et vingt-huit fragments). Il est également à l'origine de la grande majorité des textes en français sur la matière troyenne qui ont circulé au Moyen Âge.

Contrairement à la légende qui le fait inventer de l'autre côté des remparts par l'un ou l'autre chef grec, le jeu d'échecs n'est pas alors mis au point pour lutter contre l'ennui provoqué par la longueur du siège, mais bien plus tôt, à l'issue de la reconstruction de la ville par Priam, dans un moment de joie et de célébration: la réédification de la cité et la réorganisation $\mathrm{du}$ royaume sont couronnées par cette invention, avec laquelle Benoît termine sa description de la nouvelle Troie.

Les échecs y sont inventés en même temps que le jeu de dés et celui des tables ${ }^{5}$ : les jeux figurent donc, parallèlement à la réorganisation spatiale et politique de la cité, au sein des merveilles artistiques et intellectuelles que produit alors la civilisation troyenne, exemple même du raffinement courtois, et en constituent le point d'aboutissement. Ils symbolisent également l'organisation parfaite de la société troyenne.

\footnotetext{
${ }^{4}$ Léopold Constans, Le Roman de Troie par Benoît de Sainte-Maure publié d'après tous les manuscrits connus, Paris, Firmin Didot, 1904-1912.

${ }^{5}$ Pour une description des différents jeux pratiqués au Moyen Âge, $c f$. l'exposition de la BnF, dirigée par Ève Netchine, «Jeux de princes, jeux de vilains» (http://expositions.bnf.fr/jeux/) et particulièrement pour le jeu des tables, http://expositions.bnf.fr/jeux/arret/02.htm.
} 
L'espace social, dont les jeux sont le reflet ${ }^{6}$, est, comme la cité, ordonné et hiérarchisé.

Le but premier des jeux est de se deporter (« se distraire», v. 3177, 3186), mais le mot doit être entendu sans connotation péjorative, car il s'agit d'une distraction noble et courtoise, dans tous les sens du terme (v. 3172-3186) :
«Quant Ylion fu achevez
E Troie la riche citez,
Grant joie orent, mout furent lié ;
Mout ont as deus sacrefiié.
Gieus establirent e troverent,
Ou mainte feiz se deporterent ;
Onques ne fu riche maistrie
N'afaitemenz ne corteisie,
Dont l'om eüst delit ne joie,
Que ne trovassent cil de Troie :
Eschec e tables, gieu de dez
I furent, ço sacheiz, trovez,
E mainte autre uevre deportable
Riche e vaillant e delitable. »

Cette inscription courtoise transparaît par ailleurs dans une légère incohérence du texte : lors du récit de la conquête de la Toison d'or qui ouvre le Roman car elle mènera à la première destruction de Troie, des chevaliers jouent aux échecs dans le palais d'Oëtés au moment où les Argonautes débarquent (v. 1185-1192):

« Devant la sale de la tor,

Fors des arvous del parleor,

Ot une place grant e lee,

De haut mur tote avironee ;

Le trait durot a un archier :

\section{La joërent maint chevalier}

\footnotetext{
${ }^{6}$ Comme le note l'exposition déjà citée de la $\mathrm{BnF}$, « de nombreux jeux sont la mémoire d'une aspiration à l'ordre, au classement et à l'ordonnancement». Dans le cas des échecs, la hiérarchie informe les règles mêmes du jeu : les possibilités de déplacement y distinguent les pièces nobles des autres pièces. " Le jeu renouvelle à tout instant l'image d'une société hiérarchisée dans laquelle chaque membre, à sa place, garantit la cohésion de l'ensemble ».
} 


\section{As dez, as eschès e as tables, $E$ as autres gieus deportables. »}

Malgré cette légère contradiction, les échecs apparaissent aussi emblématiques du raffinement de la civilisation troyenne que la Chambre de Beauté.

Le jeu d'échecs est cependant, chez Benoît, porteur d'une certaine ambiguïté : s'il constitue une activité de cour qui démontre l'intelligence et l'éducation de ceux qui la pratiquent, il peut être utilisé à mauvais escient et devenir le signe de l'oisiveté ou du mauvais comportement d'un personnage.

Si le quinzième bâtard de Priam, Doglas, excellent joueur d'échec («Nus hom ne saveit plus d'eschas », v. 8124), est décrit positivement comme un chevalier courtois et bien éduqué, il n'en demeure pas moins que, dans le roman, c'est à Achille, personnage négatif qui illustre notamment la folie d'amour, que sont associés les échecs de la manière la plus frappante. Tombé amoureux de Polyxène, il essaye de faire lever le siège afin d'obtenir en retour la main de cette dernière. Devant le refus des Grecs, il décide de se retirer des combats; les Grecs, dans la bataille suivante, sont nettement dominés, au point que les Troyens parviennent à incendier une partie de leurs vaisseaux, tandis qu'Achille se trouve dans sa tente, jouant aux échecs. C'est alors qu'Heber, fils du roi de Trace, blessé à mort, vient le supplier de prendre les armes, et expire devant lui (v. 1907119078) :

« Achillès fait chiere e semblant

Que lui n'en seit ne tant ne quant.

N'i respont mot ne n'i entent :

O uns eschas d'or e d'argent

Joë a un chevalier des suens ;

Pense qu'ancore avra ses buens,

Quar Greu feront par estoveir

Trestot son buen e son voleir. ॥ 
Les échecs, pierre finale de la reconstruction et de la réorganisation troyenne, symboles de l'excellence de leur civilisation, peuvent donc aussi être associés à des comportements déshonorables.

\section{Les mises en prose du Roman de Troie}

Les cinq mises en prose du $\operatorname{Roman}^{7}$, si elles mentionnent l'invention des jeux, ne leur accordent pas toutes la même importance.

La première prose, qui suit généralement Benoît en l'abrégeant, dissocie la découverte des jeux de la reconstruction de la cité ; l'invention y est bien postérieure à la réédification de la ville, mais elle ne lui semble plus liée $(\mathrm{BnF}$, fr. 1612, fo 11c) :

«Aprés fu la ville puplee de la gent dou païs environ si richement que nule ville ne le resambla ne ne fu onques de son pooir. Et jeu de tables et de eschas i furent premierement trovés, et mains autres delis dont li Troien de la ville se desduisoient. »

Même chose dans Prose 4 ( $(7)$, qui met de plus l'accent sur l'abondance régnant à Troie et le bien-être qui en découlent et ancre ainsi nettement les jeux du côté des divertissements et des plaisirs. S'ils sont toujours un signe de la supériorité troyenne, c'est la prospérité de la cité qui est mise en avant plus que ses développements culturels et intellectuels ; les jeux n'ont de même plus autant d'implications sociales :

«Et si comme dit Daires, qui volentiers ne ment pas, que en tout le monde ne poïst l'en pas autretant de bones choses trouver comme l'en feist en la cité de Troie, car quancque l'en pooit deviser de boche qui a home et a fame feust necessaire a vivre et aezier soi, de tout i avoit grant plenté ne nus n'en avoit dizete.

Lors commencierent a faire festes et geuz por aux deduire et esbatre ; et, si comme pluseur dient, cil de Troie [124b] de leur joliveté trouverent le jeu des eschas, des tables et des dez. $\rangle^{8}$

\footnotetext{
${ }^{7}$ Ces dernières s'échelonnent entre le milieu du XIII ${ }^{\mathrm{e}}$ siècle et le début du XIV ${ }^{\mathrm{e}}$ siècle, $c f$. Marc-René Jung, La Légende de Troie en France au Moyen Âge, Bâle/Tübingen, Francke Verlag, 1996, p. 440-562. Nous n'évoquerons pas ici Prose 2.

${ }^{8}$ Françoise Vielliard, Le Roman de Troie en prose (version du Cod. Bodmer 147), Cologny/Genève, Fondation Martin Bodmer, 1979.
} 
Prose 3 et Prose 5, qui présentent un texte commun à cet endroit, développent au contraire le passage. La découverte du jeu y constitue, comme dans Benoît, l'ultime épisode de la reconstruction, mais la nouveauté réside dans un développement relatif au jeu de dés, inventé ensuite, sans doute lors du siège, et ajouté aux deux autres jeux. Ce dernier détail correspond à la réalité médiévale où certaines parties d'échecs se jouaient à l'aide de dés. La particularité des deux proses est que ces jeux sont devenus de véritables régulateurs des opérations militaires, en s'appuyant sur une pratique qui s'apparente à la divination (Rouen, BM, $\left.033, f^{\circ} 10 d-11 a\right)$ :

«Et en tant comme ilz furent en paix, en grant subtiveté et par grant sapience controuverent le gieu des eschecs et celui des tables sans lez déz. Celui des déz fu controuvé par grant piece aprés en celle cité mesmes: si le trouverent pucelles et damoiselles amoureuses. Et si fu ce gieu trouvé pour sortir de leur amis, non pas pour jouer, car ilz sortissoient des jours qu'ilz devoient aler en bataille pour savoir, s'ilz y aloient, s'ilz estoient en peril de mort ou non. Et cilz, s'elles veoient qu'il fussent en peril, se remanoient avecquez [11a] elles, car elles les retenoient plus pour ce que es déz quarréz venoit de aventure une pointure ou autre. Se prirent les nobles hommes es chambres en segré avecquez les damoisellez a jouer a plus poins tant que tout fu cogneu le gieu et sceu. De celui se prindrent aux autres et si adjousterent les déz aux tables et aux eschéz pareillement. »

Dans Prose 5, cet extrait est de plus directement précédé d'un ajout par rapport à Benoît comme à Prose 3 où l'organisation militaire et hiérarchique de la cité est soigneusement décrite : depuis Ilion, cœur de la ville et demeure de la famille royale, s'établissent en cercles successifs les différents niveaux de la société troyenne jusqu'aux rempart où sont installés des barons et leur suite pour surveiller les sept portes ouvrant sur les sept royaumes vassaux de Troie. Parallèlement à cette organisation spatiale est 
inventée une nouvelle organisation sociale du temps et des divertissements avec la découverte des jeux.

Les quatre proses conservent par ailleurs l'épisode où Achille joue aux échecs alors que les Grecs, en déroute, viennent le solliciter ${ }^{9}$. Le jugement péjoratif porté sur ce comportement est accentué par Prose 5 qui souligne le motif en l'évoquant à deux reprises.

Prose 3 présente enfin une autre particularité : Achille n'y est plus le seul à être représenté en train de jouer aux échecs, et on peut voir Hector s'y adonner également. Le texte de Prose 3, à cet endroit du récit, est totalement autonome de celui des autres proses. Lors du débarquement des Grecs à Troie, qui donne lieu à une grande bataille, Hector se trouve dans la Chambre de Beauté où il joue aux échecs. Entendant le bruit des combats, il jette alors l'échiquier pour prendre les armes (Rouen, BM, 0 33, $\left.f^{\circ} 26 a\right)$ :

«Hector oÿt le cry et la noise de la Chambre de Beauté ou il jouoit aux eschés, et se demanda que c'estoit. Et on lui a dit et compté que les Gregois sont arrivéz et ont a force le port prins pour assaillir leur ennemis, et que l'estour et la bataille est ja grant et efforcié sur le rivage de la mer. Hector boute suz l'esciquier et demande ses armes... ».

Cette scène constitue un contrepoint à l'attitude d'Achille: Hector, bien pourvu de toutes les qualités que doit posséder un chevalier courtois, maîtrise les échecs, mais il n'utilise pas le jeu comme support d'un comportement contraire à ses devoirs, à l'inverse d'Achille.

\footnotetext{
9 Prose 1, BnF, fr. 1612, fo 80b ; Prose 3, Rouen, BM, 0 33, fo 58b ; Prose 4, § 36 ; Prose 5, § 281 et 285 (édition Anne Rochebouet, «D'une pel toute entiere sans nulle cousture: la cinquième mise en prose du Roman de Troie, édition critique et commentaire », thèse de doctorat Université de Paris IV-Sorbonne); voir aussi l'illustration 2.
} 


\section{L'Historia destructionis Troiae de Guido delle Colonne et ses tra- ductions}

Quand il rédige à la fin $\mathrm{du}_{\mathrm{XIII}}^{\mathrm{e}}$ siècle son Historia destructionis Troiae, Guido delle Colonne ne cite pas le Roman de Troie de Benoît de Sainte-Maure comme source de son ouvrage. Et pourtant, il lui en emprunte de larges passages, plus ou moins librement traduits en latin ${ }^{10}$.

Le texte de Benoît se trouve largement amplifié chez Guido. Si l'on retrouve les jeux d'échecs, de dés et des tables, s'y ajoutent ce que nous identifierions comme des spectacles avec le théâtre ou les jeux du cirque et les festivals. Il s'agit vraisemblablement d'une amplification érudite, incluant aux jeux traditionnels des pratiques antiques bien identifiables :

«Dans cette cité ils établirent diverses sortes de jeux qu'ils y inventèrent. C'est là que furent d'abord inventés les plaisirs captivants des échecs, là les jeux de hasard (alearum), sources de colères subites, là encore les jeux de dés (taxillorum), sources de pertes soudaines et de gains éphémères. C'est là que furent d'abord institués, dit-on, tragédies et comédies, bien que certains affirment que c'est dans l'île de Sicile que fut d'abord inventée la comédie. On peut lire que c'est là que furent inventés les jeux du cirque et les fêtes du mois de mai (maiuma), qui se déroulaient selon la coutume au début de ce mois, c'est-à-dire au début du printemps, quand les arbres se couvrent de feuilles verdoyantes et que les fleurs commencent à s'épanouir. C'est là que furent inventées de nombreuses sortes d'autres jeux, qui avaient coutume de charmer l'âme des hommes et d'amener la satisfaction du plaisir sur le visage des hommes pour le plus grand plaisir des spectateurs. ${ }^{11}{ }^{\prime \prime}$

Le texte de Guido delle Colonne connaît en France dans les siècles qui suivent un large succès comme en témoignent les nombreuses œuvres auxquelles il donne naissance : Marc-René Jung dénombre ainsi cinq traductions et trois adaptations ${ }^{12}$. Parmi ces textes, tous ne conservent pas le

\footnotetext{
${ }^{10}$ Marc-René Jung, La Légende de Troie en France au Moyen Âge, op. cit., p. 563-569.

${ }^{11}$ Guido de Columnis, Historia destructionis Troiae, Nathaniel Edward Griffin (éd.), Cambridge, Massachusetts, The mediaeval academy of America, 1936, New York, Kraus Reprint co., 1970, p. 49 (c'est nous qui traduisons).

${ }^{12}$ Ibid., p. 570-601 et p. 602-613.
} 
passage consacré aux jeux dans son intégralité. Des difficultés de traduction liées au lexique peuvent expliquer son fréquent abrégement ${ }^{13}$. On s'arrêtera ici, plutôt que sur la deuxième traduction de Guido (« La furent trouvez pluiseurs jeux comme le jeu des eschés, le jeu de table et des autres pluiseurs », Bruxelles 9571-72, $\mathrm{f}^{\circ} 18 \mathrm{r}^{\circ}$ ) ou sur L'Histoire des Neuf Preux et des Neuf Preues de Sébastien Mamerot («La se trouverent premierement le jeu des tables et aultres diverses manieres de jeuz », ÖNB cod. 2577, $\mathrm{f}^{\circ} 96 \mathrm{r}^{\circ}$ ), sur une traduction (Guido A) et une adaptation (Le Triomphe des Neuf Preux) qui permettent de considérer les variations introduites par le travail du traducteur/adaptateur; on pourra ainsi déterminer la conception des jeux qui en découle et le sens à attribuer à de tels changements dans l'économie de ces œuvres créées les unes par rapport aux autres.

Guido $\mathrm{A}$, réalisée à la fin $\mathrm{du} \mathrm{XIV}^{\mathrm{e}}$ siècle, est la plus ancienne des traductions et présente des caractéristiques particulières (BnF fr. 22553, $\left.\mathrm{f}^{\circ} 22 \mathrm{v}^{\circ}\right)^{14}$ :

«Et adonc en celle cité des nobles et puissans la oyseusement habitans furent trouvéz nouveaulx jeux et ordonnéz, car la fut premierement trouvé le soubtil jeu des eschéz, le soubdain et courtsault jeu des eschéz et de tables, les hastives pertes ou anelé a queste des déz. Et la furent trouvéz les rimeurs et chanteurs de geste et faiseurs de motéz et de chansons, combien que aucuns dient que li premier chanteur en place vindrent de Cecille. Aussi furent la trouvees danses et karoles et aultres jeux qui ne sont pas en usaige orendroit, et especiaument aucunes jennes gens s'assembloient ou mois de may quant le boys est plain de fleurs et de feuillie et que raverdist la belle prayerie. Et ces jeux si faisoient pour deduire a jours de festes et tous les jours ceulx qui oyseulx estoient. »

\footnotetext{
${ }^{13}$ Un examen de la tradition manuscrite du texte latin n'a pu être réalisé étant donné le nombre extrêmement important de manuscrits renfermant ce texte; sans cet examen toutefois, on ne peut exclure totalement l'hypothèse que certains textes latins abrégeaient déjà ce passage.

${ }^{14}$ En l'absence d'étude des manuscrits et de la transmission des traductions de Guido, les manuscrits choisis pour les transcriptions l'ont été pour des raisons pratiques d'accessibilité du texte.
} 
$\mathrm{Au}$ début et à la fin de ce passage, le traducteur introduit un jugement en faisant naître les jeux de l'oisiveté. Or l'otiositas était considérée, selon Isidore de Séville repris par Thomas d'Aquin ${ }^{15}$, comme l'une des filles de l'acédie, l'un des sept péchés capitaux. La seconde spécificité de ce texte réside dans la traduction choisie pour rendre compte des spectacles théâtraux, festivals et jeux sportifs. Deux possibilités peuvent être envisagées : soit le traducteur lui-même n'a pas très bien compris le texte (le terme de maiuma par exemple est particulièrement flou), soit il a voulu adapter des concepts étrangers ou peu familiers pour le public auquel était destinée l'œuvre. Dans les deux cas, l'auteur fait le choix d'une réalité familière qui conserve la dimension de déclamation et de spectacle, tout en précisant l'étrangeté de ces coutumes.

L'auteur du Triomphe des Neuf Preux ${ }^{16}$ modifie et développe encore le passage en ajoutant de nombreuses précisions absentes du texte de Guido. On observe d'abord deux disparitions, celle des jeux de dés et celle des comédies. La seconde est peut-être liée à la difficulté d'adaptation du concept de comédie à l'époque, comme nous l'avons déjà constaté dans Guido A. Par ailleurs, les ludi circenses et maiuma donnent lieu à une vaste amplification, puisque sont ici décrits les «jeux circensés », le « jeu des gladiateurs $»$ et les $«$ Olimpiades $»{ }^{17}$ :

«Tandis que le roy Prian ediffioit sa cité en la maniere cy dessus touchee, Hector, qui ne savoit perdre temps ne vouloit donner a oyseuse ses membres, institua et pensa a soy mesmes de faire plusieurs jeux et esbatemens a plusieurs et divers propos, et selon aussi les temps et saisons ausquelz ilz se devoient jouer a cause de recreer les ouvriers qui illec continuelement labouroient. Il trouva le jeu des eschés pour en temps de pluye et d'yver solacier les chevaliiers et nobles

\footnotetext{
15 Saint Thomas d'Aquin, Summa Theologiae, Cambridge, Cambridge University Press, 2006, volume 35 (2a2æ. 34-46), Consequences of Charity, 35, 4, p. 32.

${ }^{16}$ Richard Trachsler, Clôtures du cycle arthurien, Genève, Droz, 1996, p. 296 ; MarcRené Jung, La Légende de Troie en France au Moyen Âge, op. cit., p. 610-613.

${ }^{17}$ Le Triomphe des Neuf Preux, imprimé par Pierre Gerard, Abbeville, 1487, $\mathrm{f}^{\circ} \mathrm{pVII} \mathrm{r}^{\circ}$.
} 
hommes, et le jeu de tables pour les communes gens entretenir. Aux clers fist jouer les tragedies devant le peuple, affin de oÿr raconter les gestes et avenues passee. Il institua pour les fors et robustes hommes les jeux circensés, qui estoit de combatre en une ronde place comme en ung cercle, jusques a vaincre l'un l'autre. Ce jeu se faisoit le premier jour de may, quant les arbres et les fleurs sont verdoians. Il fist et trouva pour les josnes hommes excerciter aux armes le jeu des gladiateurs que les Rommains eurent depuis en grande recommandacion. Ce jeu se faisoit d'hommes en leurs pourpoins, chascun une longue espee entre ses mains, enclos en ung cercle large, tout fermé d'espees, les pointes vers les combateurs, et illec s'esprouvoient jusques au rendre et bien souvent a la mort. Puis, et tout dernierement, institua les Olimpiades, qui estoit ung jeu qui se faisoit a courir a plutost venir a certaine enseigne ou a luitter, a ruer la barre de fer ou a saillir ou a monter le plus legierement dessus le cheval en courant. A veoir ces jeux quant ilz se faisoient venoient gens de toutes nacions du monde. Et mesmes y vint Paris...»

La structuration sociale et temporelle déjà visible chez Guido est ici accentuée par l'ajout systématique d'explications concernant l'époque où le jeu doit avoir lieu, le type de participants ou encore les règles de son déroulement. À une répartition par groupe social, chevaliers et nobles hommes s'opposant aux communes gens, les clercs aux jeunes hommes, s'ajoute une distribution en fonction de la saison et du temps : les échecs sont ainsi réservés pour l'hiver et le « temps de pluye », tandis que les jeux qui se déroulent en extérieur ont logiquement lieu à la belle saison. Enfin, chaque jeu est associé à une fonction dans la cité. Si les échecs et le jeu des tables contribuent à éviter l'inaction causée par le mauvais temps lors de la construction de Troie, les tragédies remplissent un rôle civique et politique en permettant de perpétuer la mémoire du passé, tandis que les jeux de cirque et du stade servent à entraîner aux armes la jeunesse. Les jeux ne sont pas de vaines occupations, résultant de l'inaction des Troyens, leur invention est au contraire la marque ultime de leur supériorité : c'est pour éviter de «donner a oyseuse ses membres » et en récompense des efforts fournis que sont créés les jeux. 
L'image qui apparaît alors est celle d'une cité encore plus harmonieuse que celle que décrit Guido, l'auteur allant même jusqu'à gommer tout élément discordant. La disparition des dés, décrits par l'hypotexte latin comme une source de troubles - on rejoint là la condamnation traditionnelle, dès l'Antiquité, des jeux de hasard - est vraisemblablement liée au désir de supprimer tout élément négatif qui viendrait perturber la cité idéale. L'objectif de l'auteur du Triomphe des Neuf Preux n'est pas de raconter l'histoire de Troie, mais celle d'Hector, ce qui justifie l'innovation majeure de ce passage où l'invention des jeux lui est attribuée. À travers cette cité parfaite, c'est Hector qui se trouve valorisé. Ce rapport privilégié entre la cité et son prince, s'il est traditionnel, est pourtant accentué par l'auteur du Triomphe qui déclare la supériorité d'Hector, défenseur de sa cité, sur les autres preux conquérants $^{18}$.

À travers la circulation du motif du jeu d'échecs de texte en texte, des mises en prose aux traductions et compilations, apparait dans toutes ses variations le travail de relecture et de réécriture auquel s'est prêté le Moyen Âge, notamment avec la matière troyenne. Jeu courtois par excellence, il ne pouvait avoir été créé que dans la cité idéale qu'est Troie, emblème de la civilisation et de la courtoisie. Chaque texte offre sa propre réinvention du mythe, et de ses implications, à un lectorat qui, à en croire le nombre

${ }^{18}$ Ibid., $\mathrm{f}^{\circ} \mathrm{pV}:$ « Je veuil commencer a escripre les fais et grant proesses du puissant et preux Hector, miroir de toute chevalerie et preudommie. Et ne desplaise a aucun de ce que tant de louenge luy ay desja attribuee, attendu que j'ay promis ne me rendre parcial, mais en la louenge de cestuy je ne derogue pas a la glore des autres qui tant ont fait de proesses que la terre en est plaine, et qu'ilz sont dignes et ont deservi estre portez par dame renommee par tous les siecles, ilz ont gaingné en acquerant terres et seigneuries voisines et estranges. Et cestuy Hector l'a acquise en deffendant sa propre terre et ses subgetz, et non seulement de sa force et puissance corporelle, mais ancores par donner bon conseil et prudent, se son pere et freres l'eussent voulu croire et entendre, ce qu'ilz ne firent, et dont a tart s'en vindrent a repentir, comme au long est contenu en la totale et entiere histore de Troye. » 
extrêmement important de manuscrits que représentent tous ces textes, ne se lassait pas de revisiter l'apogée et la chute de Troie.

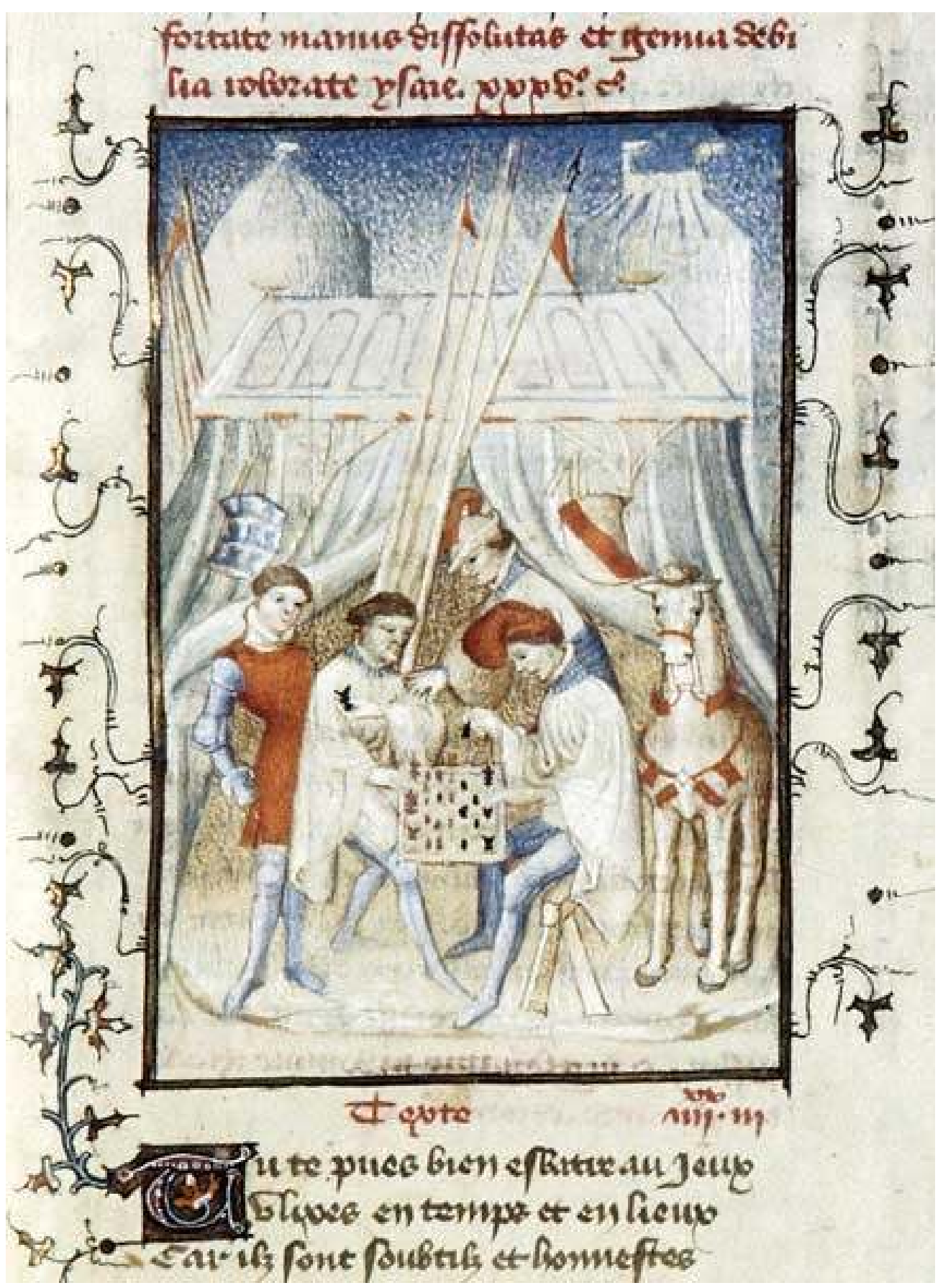

Illustration 1 : Christine de Pizan, Epitre Othea, $\mathrm{xv}^{\mathrm{e}}$ siècle, BnF, fr. $606, \mathrm{f}^{\circ} 39$. 


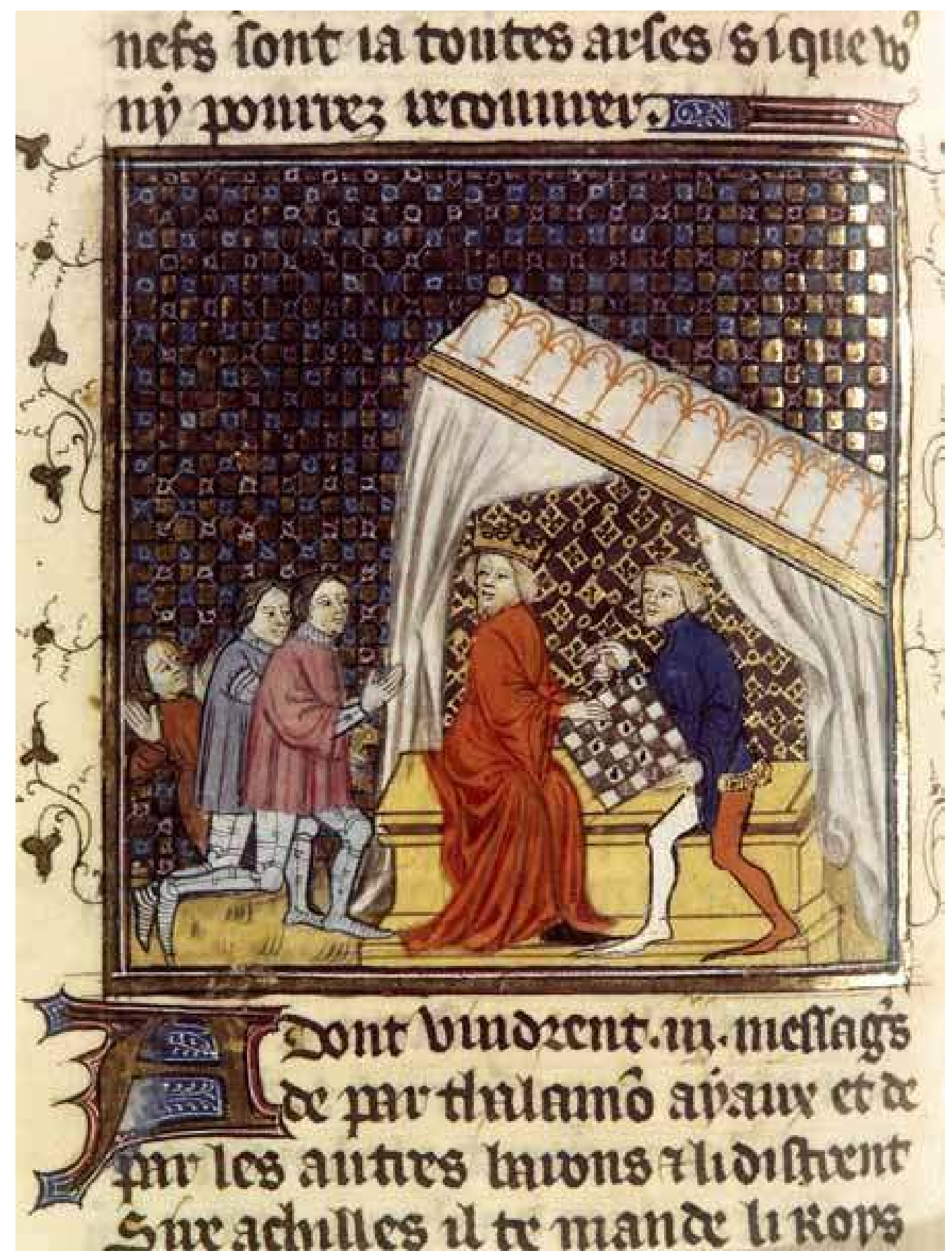

Illustration 2: Prose 5, BnF, fr. 301, f $\mathrm{f}^{\circ} 111 \mathrm{v}^{\circ}$. 